\title{
Variaciones de la composición bioquímica del fluido folicular de la alpaca según el estado de crecimiento del folículo ovárico
}

\author{
Variations of the biochemical composition of the alpaca follicular according \\ to the growth status of the ovarian follicle
}

\author{
Joel Iván Pacheco ${ }^{1,3}$, Pedro U. Coila², Macoly Olivera ${ }^{2}$, Andrés Quispe ${ }^{2}$
}

Resumen

Se determinaron las concentraciones de proteínas totales, glucosa, colesterol, triglicéridos, fosfolípidos, calcio, fósforo, magnesio, fosfatasa alcalina (ALP), aspartato transaminasa (AST), alanina transaminasa (ALT) y 17- $\beta$-estradiol en el líquido de folículos ováricos de alpacas Huacaya vacías en edad reproductiva de acuerdo con el desarrollo del folículo ovárico (tamaño). Se utilizaron 60 alpacas beneficiadas en el camal local y se tomaron muestras de folículos pequeños $(<4 \mathrm{~mm})$, medianos $(5-7 \mathrm{~mm})$ y grandes $(>7 \mathrm{~mm})(\mathrm{n}=20$ por tamaño). El diámetro de los folículos se midió con un calibrador vernier y el líquido folicular fue aspirado. Los análisis de las muestras se realizaron en el Laboratorio de Bioquímica de la Universidad Nacional del Altiplano (Puno, Perú) utilizando técnicas colorimétricas y espectrofotométricas. Los resultados de concentración para los folículos pequeños, medianos y grandes fueron: proteínas totales $(5.03,5.68$ y $7.25 \mathrm{~g} / \mathrm{dl})$, glucosa $(45.8,56.4$ y $69.0 \mathrm{mg} / \mathrm{dl})$, colesterol $(3.96,5.33 \mathrm{y}$ $6.18 \mathrm{mg} / \mathrm{dl})$, triglicéridos $(17.91,14.91$ y $12.0 \mathrm{mg} / \mathrm{dl})$, fosfolípidos $(8.99,6.0$ y $6.11 \mathrm{mg} / \mathrm{l})$, calcio $(5.76,8.19$ y $9.58 \mathrm{mg} / \mathrm{dl})$, fósforo $(15.69,10.61 \mathrm{y} 10.71 \mathrm{mg} / \mathrm{dl})$, magnesio $(2.91,2.85$ y $2.59 \mathrm{mg} / \mathrm{dl}), \operatorname{ALP}(84.6,75.4$ y $75.59 \mathrm{UI} / \mathrm{l}), \operatorname{AST}(238.17,174.83$ y $152.06 \mathrm{UI} / \mathrm{l})$, ALT $(24.24,18.0$ y $11.72 \mathrm{UI} / \mathrm{l})$ y $17-\beta$-estradiol $(0.80,1.91$ y $2.39 \mathrm{ng} / \mathrm{ml})$. Se concluye que los niveles de calcio se incrementan y los niveles de magnesio y fosfatasa alcalina disminuyen conforme se desarrolla el ovocito, mientras que los niveles de fósforo y fosfolípidos disminuyen de folículos pequeños a folículos medianos, para luego incrementarse ligeramente en folículos grandes. Además, el contenido de estradiol está en relación directa con su tamaño.

Palabras clave: alpaca; fluido folicular; composición bioquímica; crecimiento folicular

\footnotetext{
${ }^{1}$ Estación del Centro de Investigación IVITA-Maranganí, Facultad de Medicina Veterinaria, Universidad Nacional Mayor de San Marcos, Lima, Perú

${ }^{2}$ Facultad de Medicina Veterinaria y Zootecnia, Universidad Nacional del Altiplano, Puno Perú

${ }^{3}$ E-mail:jpachecoc@unmsm.edu.pe
} 
The concentrations of total proteins, glucose, cholesterol, triglycerides, phospholipids, calcium, phosphorus, magnesium, alkaline phosphatase (ALP), aspartate transaminase (AST), alanine transaminase (ALT) and 17- $\beta$-estradiol in the follicular fluid were determined in non-pregnant Huacaya alpacas in reproductive age according to the development of the ovarian follicle (size). In total, 60 slaughtered alpacas in the local slaughterhouse were used. Samples of small $(<4 \mathrm{~mm})$, medium $(5-7 \mathrm{~mm})$ and large $(>7 \mathrm{~mm})$ follicles were taken $(\mathrm{n}$ $=20$ by size). The diameter of the follicles was measured with a vernier and the follicular fluid was aspirated. The samples were analysed in the Biochemistry Laboratory of the Universidad Nacional del Altiplano (Puno, Peru) using colorimetric and spectrophotometric techniques. The concentration results for small, medium and large follicles were: total proteins $(5.03,5.68$ and $7.25 \mathrm{~g} / \mathrm{dl})$, glucose $(45.8,56.4$ and $69.0 \mathrm{mg} / \mathrm{dl})$, cholesterol $(3.96,5.33$ and $6.18 \mathrm{mg} / \mathrm{dl})$, triglycerides $(17.91,14.91$ and $12.0 \mathrm{mg} / \mathrm{dl})$, phospholipids $(8.99,6.0$ and $6.11 \mathrm{mg} / \mathrm{l})$, calcium $(5.76,8.19$ and $9.58 \mathrm{mg} / \mathrm{dl})$, phosphorus $(15.69,10.61$ and $10.71 \mathrm{mg} / \mathrm{dl})$, magnesium (2.91, 2.85 and $2.59 \mathrm{mg} / \mathrm{dl}), \operatorname{ALP}(84.6,75.4$ and $75.59 \mathrm{IU} / \mathrm{l}), \operatorname{AST}(238.17,174.83$ and $152.06 \mathrm{IU} / \mathrm{l})$, ALT $(24.24,18.0$ and $11.72 \mathrm{IU} / \mathrm{l})$ and $17-\beta$-oestradiol $(0.80,1.91$ and $2.39 \mathrm{ng} /$ $\mathrm{ml})$. It is concluded that calcium levels increase, and levels of magnesium and alkaline phosphatase decrease as the oocyte develops, while phosphorus and phospholipid levels decrease from small follicles to medium follicles, then slightly increase in large follicles. Moreover, oestradiol content is directly related to its size.

Key words: alpaca; follicular fluid; biochemical composition; follicular growth

\section{INTRODUCCIÓN}

El fluido folicular contiene compuestos importantes en el aspecto fisiológico, bioquímico y metabólico de la maduración nuclear y citoplásmica del ovocito, y experimenta notables cambios durante el ciclo estrual (Hafez E y Hafez B, 2000). La composición del líquido folicular ha sido determinada en varias especies domésticas, incluyendo el bovino (Wise, 1987; Iwata et al., 2004), ovinos (Nandi et al., 2007) y porcinos (Schuetz y Anisowicz, 1974; Chang et al., 1976). En alpacas se observan leves diferencias entre etapas de crecimiento, encontrándose una mayor cantidad de proteínas totales en folículos secundarios en comparación a folículos terciarios; asimismo, la albúmina es la proteína de mayor proporción (Aitken, 1997; Pacheco y Coila, 2010). Los lípidos totales disminuyen a medida que los folículos crecen y se convierten en preovulatorios (Pacheco y Coila, 2010).
El calcio juega un rol importante en la regulación gonadotrópica de la esteroidogenesis ovárica en ovinos, incrementándose su concentración a través del crecimiento folicular (Carnegie y Tsang, 1984; Nandi et al., 2007), mientras que la cantidad de calcio en líquido folicular de marranas es independiente del tamaño folicular (Schuetz y Anisowicz, 1974). El calcio se acumula en las células del cúmulo, pero disminuye durante la maduración ovocitaria (Boni et al., 2002). Por otro lado, los niveles de fósforo en el ovino se incrementan con el aumento del tamaño folicular, mientras que la alta concentración de magnesio en folículos pequeños podría estar vinculada con la mitosis celular de las células foliculares (Nandi et al., 2007). La baja concentración de magnesio y calcio en el líquido folicular causa una disminución en la tasa de maduración ovocitaria, y además actuarían como cofactor de reacciones enzimáticas para inducir la maduración meiótica del ovocito y la regulación de la homeostasis del calcio en el zigoto (Iwata et al., 2004). 
Los fosfolípidos son componentes principales de las membranas celulares y de las lipoproteínas circulantes, donde niveles anormalmente bajos de fosfolípidos se relacionan generalmente con una nutrición deficiente o con un síndrome de mala absorción (Bloomfield, 2002; Murray et al., 2001). Las concentraciones de fosfolípidos en el líquido folicular bovino se incrementan con el tamaño folicular (Brantmeier et al., 1987).

La fosfatasa alcalina (ALP) cataliza la hidrólisis alcalina de una gran variedad de sustratos liberando fosfato inorgánico. Está ampliamente distribuida y especialmente asociada a membranas y superficies celulares (Moreno et al., 2007). La alta concentración de ALP en estadios iniciales de desarrollo folicular estaría relacionada a altos niveles de andrógenos y progesterona (Nandi et al., 2007). La concentración de ALP disminuye a medida que avanza el ciclo estral en bovinos, posiblemente por una estimulación gonadotrópica temprana (Wise, 1987). Las transaminasas son enzimas que catalizan la transferencia de grupos amino desde un aminoácido a otro cetoácido. La aspartato transaminasa (AST) y la alanina transaminasa (ALT) son las más importantes desde el punto de vista clínico (Burtis et al., 2008). Las concentraciones de 17- $\beta$-estradiol y estrógenos conjugados en plasma de alpacas se relacionan positivamente con el tamaño folicular, incrementándose a medida que los folículos maduran (Bravo, 1990).

El objetivo del presente estudio fue determinar las concentraciones de proteínas totales, glucosa, colesterol, triglicéridos, fosfolípidos, calcio, fósforo, magnesio, fosfatasa alcalina (ALP), aspartato transaminasa (AST), alanina transaminasa (ALT) y $17-\beta$-estradiol en fluido folicular de folículos ováricos (pequeños medianos y grandes) de la alpaca.

\section{Materiales y MéTodos}

Las muestras de fluido folicular fueron colectadas en el Camal Municipal del Distrito de Nuñoa, ubicado en la provincia de Melgar, Región Puno, Perú. El análisis bioquímico de las muestras fue realizado en el Laboratorio de Bioquímica de la Facultad de Medicina Veterinaria y Zootecnia de la Universidad Nacional del Altiplano, Puno.

Se utilizaron 60 alpacas hembra Huacaya vacías y en edad reproductiva (3-8 años) que estaban destinadas a beneficio. Las muestras fueron obtenidas de animales en aparente buen estado de salud, descartándose animales enfermos y con defectos reproductivos. Se aspiró el fluido folicular de 20 folículos pequeños $(<4 \mathrm{~mm})$, de 20 folículos medianos $(5-7 \mathrm{~mm})$ y de 20 folículos grandes ( $>7 \mathrm{~mm})$, tomándose una muestra por animal. Se utilizó una regla de Vernier para medir y clasificar los folículos. Las muestras fueron rotuladas y transportadas en refrigeración al laboratorio. Las muestras fueron decantadas sin ser centrifugadas, utilizándose únicamente el sobrenadante de cada tubo.

Las proteínas totales (Proteínas totales C $\left.{ }^{\circledR}\right)$, glucosa (Glicemia $\left.{ }^{\circledR}\right)$, colesterol (Colestat $\left.{ }^{\circledR}\right)$, triglicéridos (TG Color ${ }^{\circledR}$ ), fosfolípidos (Fosfatemia $\left.{ }^{\circledR}\right)$, calcio (Ca-Color $\left.{ }^{\circledR}\right)$, fósforo (Fosfatemia $\left.{ }^{\circledR}\right)$ y magnesio $\left(\mathrm{Mg}-\mathrm{Color}{ }^{\circledR}\right)$ fueron determinados por el método espectrofotométrico (Wiener Lab, 2000). La medición de la actividad enzimática de la ALP (ALP 405®), ALT (GPT/ALT®) y AST (GOT/AST $\left.{ }^{\circledR}\right)$ se hizo mediante métodos colorimétricos utilizando reactivos de Wiener Lab®. La determinación de la concentración de 17- $\beta$-estradiol se realizó mediante la técnica de ELISA (AccuDiag ${ }^{\mathrm{TM}}$ ). La cantidad de muestra por las evaluaciones de cada folículo fue de 25 a $100 \mu 1$, siguiendo las recomendaciones de cada kit.

Para el análisis de los resultados y determinar la cantidad de calcio, magnesio, fosforo, fosfolípidos y fosfatasa alcalina se 
Cuadro 1. Componentes bioquímicos en fluido folicular de alpaca

\begin{tabular}{lccccc}
\hline Tamaño folicular & $\begin{array}{c}\text { Proteína } \\
\mathrm{g} / \mathrm{dl}\end{array}$ & $\begin{array}{c}\text { Glucosa } \\
\mathrm{mg} / \mathrm{dl}\end{array}$ & $\begin{array}{c}\text { Colesterol } \\
\mathrm{mg} / \mathrm{dl}\end{array}$ & $\begin{array}{c}\text { Triglicéridos } \\
\mathrm{mg} / \mathrm{dl}\end{array}$ & $\begin{array}{c}\text { Fosfolípido } \\
\mathrm{mg} / \mathrm{l}\end{array}$ \\
\hline Pequeños & $5.03^{\mathrm{b}}$ & $45.8^{\mathrm{b}}$ & $3.96^{\mathrm{c}}$ & $17.91^{\mathrm{a}}$ & $8.99^{\mathrm{a}} \pm 0.3 \mathrm{a}$ \\
Medianos & $5.68^{\mathrm{b}}$ & $56.4^{\mathrm{b}}$ & $5.33^{\mathrm{b}}$ & $14.91^{\mathrm{b}}$ & $6.0^{\mathrm{b}} \pm 0.25$ \\
Grandes & $7.25^{\mathrm{a}}$ & $69.0^{\mathrm{a}}$ & $6.18^{\mathrm{a}}$ & $12.00^{\mathrm{c}}$ & $6.11^{\mathrm{b}} \pm 0.2{ }^{\mathrm{a}}$ \\
\hline
\end{tabular}

${ }^{a, b}$ Letras diferentes dentro de columnas indican diferencia estadística $(p<0.05)$

utilizó un diseño estadístico completamente al azar. La prueba de Tukey se usó para establecer diferencias entre medias a un nivel de significancia de 0.05 . El procesamiento de datos y el análisis estadístico se realizó utilizando el software SPSS.

\section{Resultados y Discusión}

Las concentraciones de los componentes bioquímicos del fluido folicular de alpacas según el tamaño folicular se presentan en el Cuadro 1.

La concentración de proteína en el fluido folicular fue similar a un reporte previo de los autores en folículos pequeños, observándose un incremento con la maduración de los folículos, lo cual difiere de dicho estudio donde se observó una ligera disminución de proteínas totales en folículos grandes ( $7 \mathrm{~mm}$ ) en alpacas (Pacheco y Coila, 2010). La concentración de glucosa fue similar entre folículos pequeños y medianos, siendo mayor $(\mathrm{p}<0.05)$ en folículos grandes, tal y como lo reportan Pacheco y Coila (2010). Este comportamiento posiblemente juegue un papel importante en el proceso de maduración folicular y ovulación (Murray et al., 2001); sin embargo, es diferente a lo descrito por Gerard et al. (2002) en yeguas. Es posible que también sea una fuente adicional de glucosa para los espermatozoides, puesto que una parte de este fluido ingresa hacia el oviducto después de la ovulación, toda vez que la glucosa es una fuente energética para espermatozoides de alpaca (Garnica et al., (1993, 1995).

La cantidad de colesterol en el fluido folicular de la alpaca se incrementa con la maduración del folículo evidenciando su necesidad, posiblemente como fuente primaria para la esteroidogenesis (Squires, 2003). La concentración registrada es superior a la reportado en marranas (Chang et al., 1976) y diferente a la indicada por Pacheco y Coila (2010) quienes indican que el colesterol estaría disminuyendo ligeramente a medida que los folículos maduran, pudiendo haberse debido al efecto de la centrifugación de las muestras. No existen reportes sobre concentraciones de triglicéridos en fluido folicular; sin embargo, los resultados obtenidos concuerdan con las observaciones de Pacheco y Coila (2010), donde los lípidos totales disminuyen a medida que los folículos incrementan su tamaño; así mismo, los fosfolípidos disminuyen a medida que los folículos incrementan su diámetro, como ha sido observado en vacunos, donde se reportan concentraciones de fosfolípidos en folículos pequeños, medianos y grandes de $62.7,48.3$ y $41.1 \mathrm{mg} / \mathrm{dl}$ (Brantmeier et al., 1987). Este comportamiento se debería a que los fosfolípidos y otros lípidos son utilizados para la formación de membranas celulares durante la proliferación de las células del cúmulo y la granulosa (Bloomfield, 2002). 
Las cantidades de calcio encontradas en este estudio son menores a las reportadas en ovinos (Nandi et al., 2007); mientras que se han reportado concentraciones similares en folículos pequeños $(7.6 \mathrm{mg} / \mathrm{dl})$ y en folículos grandes $(8.4 \mathrm{mg} / \mathrm{dl}$ ) en vacunos (Wise 1987 ; Iwata et al. 2004). Por otro lado, se han reportado valores superiores en cerdos (Schuetz y Anisowicz, 1974; Chang et al., 1976). Esta característica podría deberse a una singularidad en la fisiología intrafolicular del calcio en los camélidos sudamericanos, además la cantidad de calcio necesaria para la reactivación meiótica, tal como indica Boni et al. (2002). Es importante anotar, además, que calcio adicional podría provenir del fluido oviductal, en donde la concentración de calcio es superior al de otras especies (Pacheco y Olivera, 2006). Estas concentraciones de calcio son dependientes y reguladas por la presencia de $\mathrm{LH}$, que en alpacas no presenta niveles altos, salvo después de la cópula, tal como indican Bravo (1990) y Batta y Knundsen (1980).

El fósforo es necesario en los estadios tempranos del desarrollo folicular, probablemente debido a la formación de nuevas estructuras celulares, dado que una de sus funciones es servir como elemento estructural en la membrana celular (Murray et al., 2001). Esto podría explicar alta concentración de fósforo en folículos pequeños. Los valores de fósforo hallados fueron ligeramente inferiores a los reportados en ovinos (Nandi et al., 2007). Por otro lado, Chang et al. (1987) reporta concentraciones de $9.5,8.2$ y $8.1 \mathrm{mg} /$ dl en líquido folicular de marranas para folículos pequeños, medianos y grandes, respectivamente.

El magnesio desciende a medida que se incrementa el tamaño folicular (Cuadro 2). Este cambio estaría relacionado a la concentración de calcio en el líquido folicular, puesto que como el magnesio regula la actividad y concentración del calcio, se observa que la concentración de magnesio disminuye de manera inversamente proporcional al incremento de la concentración de calcio. Esto se evidencia durante el proceso de maduración ovocitaria, la cual se realiza en folículos grandes previo a la ovulación y subsiguiente fecundación, tal como los indica Iwata et al. (2004) y McDonald y Edward (1995).

La actividad de la ALP fue significativamente superior en folículos pequeños que en medianos y grandes $(\mathrm{p}<0.05$; Cuadro 3). Similar comportamiento encontró Zia-UrRahman et al. (2008) al estudiar la actividad de la ALP en fluido folicular de camellos, reportando una mayor concentración en folículos pequeños $(98.0 \mathrm{UI} / \mathrm{l})$ que en folículos grandes (92.6 UI/1). De igual forma, Nandi et al. (2007) en fluido folicular de ovinos encontraron que la actividad de la ALP fue disminuyendo significativamente a medida que el tamaño del folículo aumentaba. Mishra et al. (2003) en cabras también determinaron que la actividad de esta enzima presentaba una tendencia inversa; es decir, la actividad disminuye a medida que el folículo desarrolla.

La más alta actividad de la ALP en las fases iniciales del desarrollo folicular podría deberse al ambiente predominante en andrógenos que existen en el folículo pequeño, lo cual induce a una mayor actividad de la ALP, tal como lo indica Kalmath (2007) en búfalos. La disminución de la actividad en los folículos mayores se debería al cambio en el ambiente hormonal de los folículos en desarrollo, en donde predominan los estrógenos sobre los andrógenos (Nandi et al., 2007).

En forma similar, las actividades de las enzimas AST y ALT fueron significativamente superiores en folículos pequeños que en medianos y grandes ( $<<0.05$; Cuadro 3$)$. Zia-UrRahman et al. (2008) en fluido folicular de folículos pequeños y grandes de camellos encontraron diferencias significativas $(138.3 \mathrm{y}$ $106.9 \mathrm{UI} / 1$ para AST y 45.9 y $28.7 \mathrm{UI} / 1$ para ALT en folículos pequeños y grandes, respectivamente). Por otro lado. Iwata (2004) encontró una mayor actividad de AST en fluido folicular de bovinos en comparación con la concentración en suero sanguíneo. 
Cuadro 2. Concentración de minerales en fluido folicular de alpaca en diferentes estadios de desarrollo folicular

\begin{tabular}{lccc}
\hline Tamaño folicular & $\begin{array}{c}\text { Calcio } \\
(\mathrm{mg} / \mathrm{dl})\end{array}$ & $\begin{array}{c}\text { Fósforo } \\
(\mathrm{mg} / \mathrm{dl})\end{array}$ & $\begin{array}{c}\text { Magnesio } \\
(\mathrm{mg} / \mathrm{dl})\end{array}$ \\
\hline Pequeños & $5.76^{\mathrm{c}} \pm 0.22$ & $15.69^{\mathrm{a}} \pm 0.58$ & $2.91^{\mathrm{a}} \pm 0.08$ \\
Medianos & $8.19^{\mathrm{b}} \pm 0.33$ & $10.61^{\mathrm{b}} \pm 0.45$ & $2.85^{\mathrm{b}} \pm 0.11$ \\
Grandes & $9.58^{\mathrm{a}} \pm 0.36$ & $10.71^{\mathrm{b}} \pm 0.44$ & $2.59^{\mathrm{c}} \pm 0.10$ \\
\hline
\end{tabular}

$a, b, c$ Letras diferentes dentro de columnas indican diferencia estadística $(p<0.05)$

Cuadro 3. Concentraciones de fosfatasa alcalina (ALP), aspartato transaminasa (AST), alanina transaminasa (ALT) y estradiol en fluido folicular de alpaca

\begin{tabular}{lcccc}
\hline \multirow{2}{*}{ Tamaño folicular } & ALP & AST & ALT & 17- $\beta$-estradiol \\
& UI/l & UI/1 & UI/1 & ng/ml \\
\hline Pequeños & $84.60^{\mathrm{a}}$ & $238.17^{\mathrm{a}}$ & $24.24^{\mathrm{a}}$ & $0.80^{\mathrm{c}} \pm 0.10$ \\
Medianos & $75.40^{\mathrm{b}}$ & $174.83^{\mathrm{b}}$ & $18.00^{\mathrm{b}}$ & $1.91^{\mathrm{b}} \pm 0.18$ \\
Grandes & $75.59^{\mathrm{b}}$ & $152.06^{\mathrm{b}}$ & $11.72^{\mathrm{c}}$ & $2.39^{\mathrm{a}} \pm 0.15$ \\
\hline
\end{tabular}

$a, b, c$ Letras diferentes dentro de columnas indican diferencia estadística $(p<0.05)$

Las transaminasas son enzimas que participan activamente en el metabolismo de compuestos nitrogenados, esencialmente en el metabolismo de aminoácidos y proteínas, y nucleótidos y ácidos nucleicos; por consiguiente, la alta actividad de estas enzimas en folículos pequeños indican los altos requerimientos de aminoácidos en las fases tempranas del desarrollo folicular debido a la mayor proliferación celular de la granulosa y, sobre todo, de la teca interna del folículo (Zia-UrRahman et al., 2008). Además, también podría deberse, tal como lo indican Kalmath (2007) y Nandi et al. (2007), al ambiente hormonal que predomina en cada fase del desarrollo folicular, tal como se indicó para el caso de la ALP.

Comparando los niveles de la AST y ALT de fluido folicular de alpacas con el de camellos, especies congéneres, se observa que existen ligeras variaciones. Así por ejem- plo, la actividad promedio de la AST en alpacas es de $188.35 \mathrm{UI} / \mathrm{l}$, valor superior al del camello (122.6 UI/1), y el valor de ALT $17.98 \mathrm{UI} / \mathrm{l}$ es inferior al del camello (37.3 UI/l), atribuyendo estas diferencias a las características propias de la especie.

Por los resultados obtenidos, se demuestra que en folículos ováricos de alpacas, al igual que en otras especies, hay una mayor actividad de las enzimas ALP, AST y ALT en folículos pequeños, como resultado de la mayor demanda metabólica por energía y sustratos para el desarrollo y maduración del folículo, fruto de la mayor proliferación celular de los distintos compartimientos foliculares.

Por último, las concentraciones de 17$\beta$-estradiol fueron de $0.80 \pm 0.1,1.91 \pm 0.17 \mathrm{y}$ $2.39 \pm 0.15 \mathrm{ng} / \mathrm{ml}$ para folículos pequeños, medianos y grandes, respectivamente, incrementando visiblemente su concentración 
de acuerdo al grado de crecimiento y diámetro folicular, de acuerdo con lo descrito por Bravo (1990), y como también ocurre en otras especies, puesto que el fluido folicular se comporta como un reservorio de estrógenos, incrementándose de acuerdo al aumento de su tamaño y acercándose al momento de la ovulación (Squires, 2003).

\section{Conclusiones}

- Los niveles de proteínas, glucosa, calcio, colesterol y $17-\beta$-estradiol se incrementan a medida que los folículos maduran e incrementan su diámetro.

- Los niveles de triglicéridos, fosfolípidos, magnesio y fosfatasa alcalina disminuyen su concentración a medida que se incrementa el diámetro folicular.

- Los niveles de fósforo disminuyen de folículos pequeños a medianos, para luego incrementarse ligeramente en folículos grandes.

- Las concentraciones de minerales, fosfolípidos y fosfatasa alcalina son menores a las concentraciones obtenidas en otras especies.

\section{Literatura Citada}

1. Aitken RJ. 1997. Molecular mechanism regulating human sperm function. Mol Hum Reprod 3: 169-173. doi: 10.1093/ molehr/3.3.169

2. Batta SK, Knundsen JF. 1980. Calcium concentration in cumulus enclosed oocytes of rats after treatment with pregnant mares serum. Biol Reprod 22: 243-246.

3. Brantmeier SA, Grummer RR, Ax RL. 1987. Concentrations of high density lipoproteins vary among follicular sizes in the bovine. J Dairy Sci 70: 2145-2149.

4. Bloomfield, M. 2002. Química de los organismos vivos. México: Limusa. 200 p.
5. Boni R, Cuomo A, Tosti E. 2002. Developmental potential in bovine oocytes is related to cumulus-oocyte complex grade, calcium current activity and calcium stores. Biol Reprod 66: 836842. doi: 10.1095/biolreprod66.3.836

6. Bravo W. 1990. Studies on ovarian and response to copulation in the south American camelids, Lama glama and Lama pacos. PhD Thesis. USA: University of California. $91 \mathrm{p}$.

7. Burtis C, Ashwood E, Bruns D. 2008. Tietz fundametals of clinical chemistry. $6^{\text {th }}$ ed. Saunders. $976 \mathrm{p}$.

8. Carnegie JA, Tsang BK. 1984. The calcium-calmodulin system: participation in the regulation of steroidogenesis at different stages of granulose cell differentiation. Biol Reprod 30: 515-522. doi: 10.1095/biolreprod30.2.515

9. Chang SC, Jones JD, Ellefson RD, Ryan RJ. 1976. The porcine ovarian follicle selected chemical analysis of follicular fluid at different developmental stages. Biol Reprod 15: 321-328. doi: 10.1095/biolreprod15.3.321

10. Garnica J, Achata $R$, Bravo $P W$. 1993. Physical and biochemical characteristics of alpaca semen. Anim Reprod Sci 32: 85-90. doi: 10.1016/03784320(93)90059-Z

11. Garnica J, Flores E, Bravo PW. 1995. Citric acid and fructose concentrations in seminal plasma of the alpaca. Small ruminant Res 18: 95-98. doi: 10.1016/ 0921-4488(95)00720-6

12. Gérard N, Loiseau S, Duchamp G, Seguin F. 2002. Analysis of the variations of follicular fluid composition during follicular growth and maturation in the mare using proton nuclear magnetic resonance (1H NMR). Biol Reprod 124: 241-248. doi: 10.1530/ rep. 0.1240241

13. Hafez ESE, Hafez B. 2000. Reproducción e inseminación artificial en animales. $7^{\mathrm{a}}$ ed. México: Interamericana. $512 \mathrm{p}$.

14. Iwata H, Hashimoto S, Ohota M, Kimura K, Shibano K, Miyake M. 2004. Effects of follicle size and 
electrolytes and glucose in maturation medium on nuclear maturation and develop. competence of bovine oocytes. Reproduction 127: 159-164. doi: 10.1530/ rep. 1.00084

15. Kalmath GP, Ravindra JP. 2007. Mineral profiles of ovarian antral follicular fluid in buffaloes during follicular development. Indian J Anim Res 41: 87-93.

16. Mc Donald P, Edwards R.1995. Nutrición animal. $5^{\mathrm{a}}$ ed. Zaragoza, España: Acribia. $524 \mathrm{p}$.

17. Moreno A, González L, Mendoza-J, García-Buey L, Moreno R. 2007. Utilidad de los parámetros analíticos en el diagnóstico de las enfermedades hepáticas. An Med Intern 24: 38-46.

18. Mishra OP, Pandey JN, Gawande PG. 2003. Study on biochemical constituents of caprine ovarian follicular fluid after superovulation. Asian Austral J Anim 16: 1711-1715. doi: 10.5713/ajas.2003.1711

19. Murray R, Mayes P, Granner D, Rodwell V. 2001. Bioquímica de Harper. $15^{\mathrm{a}}$ ed. México: Manual Moderno. $692 \mathrm{p}$.

20. Nandi S, Girish V, Manjunatha BM, Gupta PS. 2007. Biochemical composition of ovine follicular fluid in relation to follicle size. Dev Growth Differ 49: 61-66. doi: 10.1111/j.1440-169X.2007.00901.x

21. Pacheco JI, Olivera L. 2006. Modificaciones estructurales de la mucosa y componentes del fluido oviductal de alpaca en fase folicular. En: XXIX Re- unión Científica Anual de la Asociación Peruana de Producción Animal - APPA. Huancayo. Perú.

22. Pacheco JI, Coila P. 2010. Composición bioquímica del fluido folicular de alpaca (Vicugna pacos) en diferentes estadios de desarrollo. Arch Zootec 59: 451-454.

23. Schuetz A, Anisowicz A. 1974. Cation and protein composition of ovarian follicular fluid of the pig: relation al follicle Size. Biol Reprod 11: 64-72. doi: 10.1095/ biolreprod11.1.64

24. Squires EJ. 2003. Applied animal endocrinology. Cambridge, USA: CABI Publishing. $234 \mathrm{p}$.

25. Wiener Lab. 2000. Catálogo de productos., Rosario, Argentina. [Internet]. Disponible en: https://www.wienerlab.com.ar

26. Wise T. 1987. Biochemical analysis of bovine follicular fluid: albumin, total protein, lisosomal enzymes, ions, steroids in relation to follicular size, rank, atresia classification and day of estrous cycle. J Anim Sci 64: 1153-1169. doi: 10.2527/ jas 1987.6441153x

27. Zia-Ur-Rahman, Bukhari SA, Ahmad N, Akhtar N, Ijaz A, Yousaf MS, Haq IU. 2008. Dynamics of follicular fluid in one-humped camel (Camelus dromedarius). Reprod Domest Anim 43: 664671. doi: 10.1111/j.1439-0531.2007.00967.x 